\title{
Ordered Semiconducting Nitrogen-Graphene Alloys
}

\author{
H. J. Xiang, ${ }^{1}$ B. Huang, ${ }^{2}$ Z. Y. Li, ${ }^{3}$ S.-H. Wei, ${ }^{2}$ J. L. Yang, ${ }^{3}$ and X. G. Gong ${ }^{1}$ \\ ${ }^{1}$ Key Laboratory of Computational Physical Sciences, Ministry of Education, and Department of Physics, \\ Fudan University, Shanghai 200433, People's Republic of China \\ ${ }^{2}$ National Renewable Energy Laboratory, Golden, Colorado 80401, USA \\ ${ }^{3}$ Hefei National Laboratory for Physical Sciences at Microscale, University of Science and Technology of China, \\ Hefei, Anhui 230026, People's Republic of China \\ (Received 23 August 2011; published 18 January 2012)
}

\begin{abstract}
The interaction between substitutional nitrogen atoms in graphene is studied by performing firstprinciples calculations. The effective nearest-neighbor interaction between nitrogen dopants is found to be highly repulsive because of the strong electrostatic repulsion between nitrogen atoms. This interaction prevents the full nitrogen-carbon phase separation in nitrogen-doped graphene. Interestingly, there are two relatively stable nitrogen-nitrogen pair configurations, whose stability can be attributed to the anisotropy in the charge redistribution induced by nitrogen doping. We reveal two stable, ordered, semiconducting $\mathrm{N}$-doped graphene structures, $\mathrm{C}_{3} \mathrm{~N}$ and $\mathrm{C}_{12} \mathrm{~N}$, through the cluster-expansion technique and particle-swarm optimization method. In particular, we show that $\mathrm{C}_{12} \mathrm{~N}$ has a direct band gap of $0.98 \mathrm{eV}$. The heterojunctions between $\mathrm{C}_{12} \mathrm{~N}$ and graphene nanoribbons might be a promising basis for organic solar cells.
\end{abstract}

DOI: 10.1103/PhysRevX.2.011003 Subject Areas: Computational Physics, Condensed Matter Physics, Graphene

\section{INTRODUCTION}

Graphene, a single layer of carbon atoms arranged in a honeycomb lattice, has been a focus of recent research efforts [1-3] due to its unique semimetallic zero-gap electronic structure and the massless Dirac-fermion behavior. The unusual electronic and structural properties make graphene a promising material for the next generation of faster and smaller electronic devices. However, graphene lacks an essential feature for controlled and reliable transistor operation, namely, a band gap around the Fermi level. Several schemes have been proposed to open a band gap in graphene. One is the substrate-induced gap for graphene supported on silicon carbide ( $\mathrm{SiC}$ ) [4], but the experimental realization of this idea turned out to be very difficult and controversial [5]. Another approach is the creation of gaps through confinement [6], such as in narrow graphene nanoribbons (GNRs). However, large-scale production of narrow GNRs is still very challenging. In addition, it has been demonstrated that the band gap of a graphene bilayer can be controlled externally by applying a gate bias $[7,8]$. Unfortunately, band-gap tailoring by an external electrostatic gate has limited tunability for the transistor on-off ratio. Chemical functionalization $[9,10]$ is an alternative way to manipulate the electronic properties of graphene. The hydrogenation of graphene [9,11-17], as a prototype of covalent chemical functionalization, has been studied extensively. However, the fully

Published by the American Physical Society under the terms of the Creative Commons Attribution 3.0 License. Further distribution of this work must maintain attribution to the author $(s)$ and the published article's title, journal citation, and DOI. hydrogenated graphene, known as graphane $\mathrm{CH}$, is an insulator with a very large band gap (larger than $5 \mathrm{eV}$ ) [18], which is unsuitable for applications in a field-effect transistor. There were some efforts to tune the band gap of two-dimensional (2D) graphene by varying the hydrogen concentration. Unfortunately, a phase separation between bare graphene and graphane takes place spontaneously, leading to an essentially zero band gap for the whole system [12]. A similar phenomenon was also found to occur in oxidized graphene [19].

Previous studies [20-25] have revealed that doping graphene and related materials with nitrogen is effective in tailoring its electronic property and chemical reactivity because of the stronger electronegativity of nitrogen compared to that of carbon and the conjugation between the nitrogen lone-pair electrons and the graphene $\pi$ system. This could create novel nanomaterials and expand the already widely explored potential applications of graphene. Experiments [26] suggest that nitrogen species have been incorporated into the graphene structure with content in the range of $4.5-16.4 \%$. Usually, the nitrogen atoms are substitutionally incorporated into the basal plane of graphene in the forms of pyridinic, pyrolic, and graphitic nitrogen bonding configurations. X-ray photoelectron spectroscopy has shown that the pyridinic and pyrolic nitrogen may lie at the edge or the defect sites, and that the graphitic nitrogens, which replace carbon atoms in the graphene plane, are dominant [27]. A recent scanning-tunneling-microscopy study has also shown that individual nitrogen atoms are incorporated in the graphitic bonding configuration [28]. Theoretically, the isolated substitutional nitrogen doping in graphene has been studied before [28]. However, the nature of the interaction between substitutional nitrogen atoms is 
far from clear. How the electronic structures of graphene are modified by multiple nitrogen dopants is still an interesting open question.

In this paper, we examine the collective behavior of nitrogen doping in graphene on the basis of densityfunctional-theory (DFT) calculations. We find that the nearest-neighbor interaction between nitrogen dopants is highly repulsive as a result of the strong electrostatic repulsion between negatively charged nitrogen atoms. Our calculations reveal two stable, ordered structures of $\mathrm{N}$-doped graphene: $\mathrm{C}_{3} \mathrm{~N}$ and $\mathrm{C}_{12} \mathrm{~N}$. Surprisingly, nitrogen atoms do not simply donate electrons to the graphene $\pi^{*}$ state. Instead, both ordered structures are found to be semiconducting. In particular, $\mathrm{C}_{12} \mathrm{~N}$ has a direct band gap of $0.98 \mathrm{eV}$. The substitutional nitrogen raises the valenceband-maximum (VBM) and conduction-band-minimum (CBM) level in $\mathrm{N}$-doped graphene, leading to a type-II band alignment between $\mathrm{C}_{12} \mathrm{~N}$ and an armchair graphene nanoribbon with six dimer lines (6-AGNR). We propose that $\mathrm{C}_{12} \mathrm{~N} / 6$-AGNR heterojunctions are a promising candidate for a new type of organic solar cell with $\mathrm{C}_{12} \mathrm{~N}$ as a donor and 6-AGNR as an acceptor, respectively.

\section{COMPUTATIONAL METHOD}

In our DFT calculations, we adopt the local-density approximation (LDA) unless otherwise stated. The planewave cutoff energy for wave function is set to $500 \mathrm{eV}$. The ion-electron interaction is treated by using the projectoraugmented-wave (PAW) [29] technique as implemented in the Vienna ab-initio simulation package [30]. For the Brillouin-zone sampling, we use $2 \times 2 \times 1$ MonkhorstPack $k$-mesh for the $12 \times 12 \times 1$ graphene supercell. And for $\mathrm{C}_{3} \mathrm{~N}\left(\mathrm{C}_{12} \mathrm{~N}\right)$, we use the $12 \times 12 \times 1(6 \times 6 \times 1)$ Monkhorst-Pack $k$-mesh. Constant-temperature firstprinciples molecular-dynamics simulations are performed to check the thermal stability of $\mathrm{C}_{3} \mathrm{~N}$ and $\mathrm{C}_{12} \mathrm{~N}$. The "cluster expansion" [31] of the alloy Hamiltonian is then carried out by using the ATAT package [32]. Then a global-minimum optimization is performed by using the two-dimensional particle-swarm-optimization (PSO) technique, which we proposed recently to predict the most stable $2 \mathrm{D}$ crystals [33]. For the PSO simulations, we use the "Crystal structure AnaLYsis by Particle Swarm Optimization" (CALYPSO) code [34].

\section{RESULTS AND DISCUSSION}

\section{A. Interaction between nitrogen dopants}

To investigate the interaction between two substitutional nitrogen atoms, we use a $12 \times 12$ supercell of graphene. The use of such a large supercell is essential due to the relatively long-range interaction between nitrogen dopants. (We note that our test calculations show that using larger supercells gives similar results.) As is well known, the honeycomb lattice of graphene consists of two interpene- trating triangular sublattices: sublattice A and sublattice B. We consider the interaction between a nitrogen atom at an arbitrarily chosen site (labeled as 0A) and those at other sites [see Fig. 1(a)]. All interactions with the interaction range of less than $6.5 \AA$ are included, and we also include several other $\mathrm{N}-\mathrm{N}$ pairs with longer-range interactions (0A-11A, 0A-12B, 0A-13B). For each N-N pair, the internal atomic structure is fully relaxed.

The calculated interaction energies of all the N-N pairs considered are plotted in Fig. 1(b) with respect to the energy of the longest-range N-N pair (0A-13B of range $15.60 \AA$ ). First, we can see that all of the interaction energies are positive, i.e., the interactions are repulsive. This can be understood as follows: Nitrogen has a higher electronegativity than carbon; consequently, nitrogen atoms are surrounded by more electrons, thus experiencing a repulsive, electrostatic interaction. Second, generally, the interaction energy decreases with the pair distance because of the nature of Coulombic electrostatic interaction. Third, the nearest-neighbor interaction is extremely repulsive, not only because the distance between them is the shortest, but also because the two nitrogen atoms are directly connected along the bond direction. Fourth, and most important, for

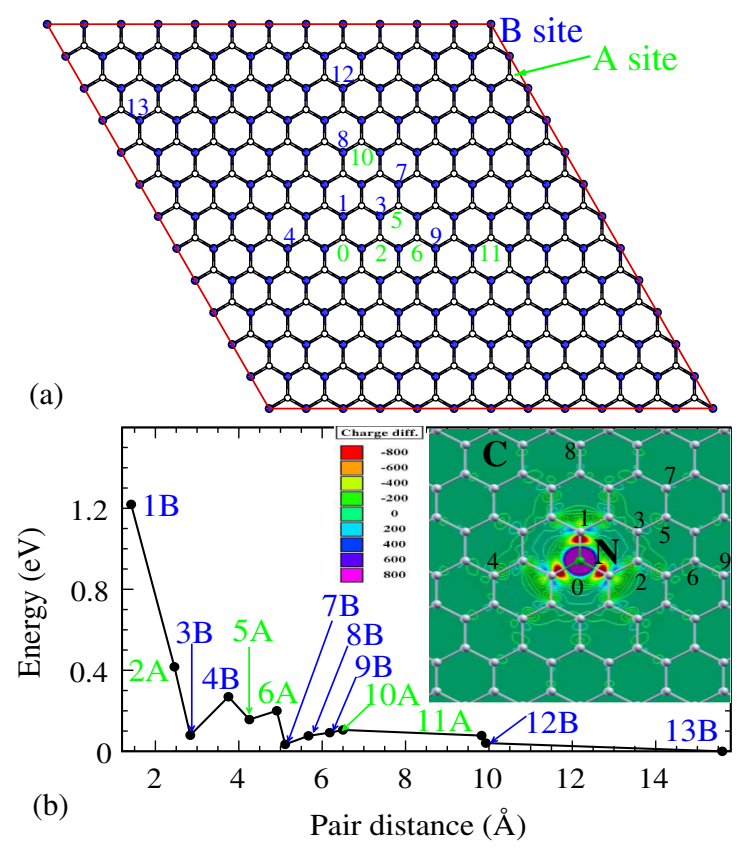

FIG. 1. (a) The $12 \times 12$ graphene supercell. The small solid (blue) or empty circles belong to sublattice A or B, respectively. The numbers label the carbon-lattice sites for nitrogen substitution. (b) The pair-interaction energy between nitrogen atoms as a function of the pair distance from the DFT total-energy calculations for the relaxed structures. For each N-N pair, one of the nitrogen atoms is at the 0 site (one of the A sites) and the other nitrogen atom is labeled in (a). The inset in (b) shows the contour plot of the electron charge-density difference between the isolated $\mathrm{N}$-doped graphene and undoped graphene. 
N-N pairs with short pair distances (less than $6.5 \AA$ ), the interaction energy curve has two local minima, at $0 \mathrm{~A}-3 \mathrm{~B}$ and $0 \mathrm{~A}-7 \mathrm{~B}$ pairs. The interactions between $0 \mathrm{~A}$ and $3 \mathrm{~B}$ and between $0 \mathrm{~A}$ and $7 \mathrm{~B}$ are only slightly repulsive, with energies of $0.08 \mathrm{eV}$ and $0.03 \mathrm{eV}$, respectively.

The nontrivial nonmonotonic dependence of the pairinteraction energy on the N-N distance and, in particular, the presence of local minima at N-N pairs $0 \mathrm{~A}-3 \mathrm{~B}$ and 0A-7B is interesting. Our calculations show that the strain relaxation is not responsible for the relative stability of the $0 \mathrm{~A}-3 \mathrm{~B}$ and $0 \mathrm{~A}-7 \mathrm{~B} \mathrm{~N}-\mathrm{N}$ pairs because the energies of the unrelaxed pair structures display the same trend. Instead, we find that the stability is due to the anisotropic distribution of the electron charges. The inset in Fig. 1(b) shows the charge difference between the graphene doped by an isolated nitrogen atom and undoped graphene. We can see that the charge redistribution in graphene takes place mainly along the nearest-neighbor bonding direction. Since sites 3B and 7B are nearly opposite to the bonding direction, there is much less charge redistribution around site $3 \mathrm{~B}(7 \mathrm{~B})$ than around $2 \mathrm{~A}$ and $4 \mathrm{~B}(6 \mathrm{~A}$ and $8 \mathrm{~B})$, respectively. Therefore, in the case of the 0A-3B and 0A-7B N-N pairs, the Coulombic repulsion will be weaker than that between the other neighboring pairs.

Our calculations show that the interaction between substitutional nitrogen atoms in graphene differs fundamentally from that between adsorbed hydrogen (oxygen) atoms on graphene. In the hydrogenated-graphene case, hydrogen atoms tend to be close to each other in order to lower the kinetic energy of the carbon $\pi$ electrons, and this tendency leads to a phase separation between fully hydrogenated graphene and bare graphene [12]. A similar phenomenon happens in the oxidized-graphene case [19]. The repulsive interaction between substitutional nitrogen atoms prevents the phase separation of nitrogen-doped graphene into one region of undoped graphene and another of highly nitrogen-doped graphene, in contrast to the case of the hydrogenation and oxidization of graphene [12,19].

\section{B. Ordered semiconducting nitrogen-graphene alloys}

In our discussion thus far, $0 \mathrm{~A}-3 \mathrm{~B}$ and $0 \mathrm{~A}-7 \mathrm{~B}$ are two relatively stable configurations of N-N pairs. Based on each configuration, we can construct a graphene superstructure with a uniform distribution of nitrogen dopants. Corresponding to the $0 \mathrm{~A}-3 \mathrm{~B} \mathrm{~N}-\mathrm{N}$ pair, the ordered nitrogen-doped graphene structure is a $2 \times 2$ superstructure $\left(\mathrm{C}_{3} \mathrm{~N}\right)$, as shown in Fig. 2(a). In this superstructure, the doped nitrogen atoms themselves form a honeycomb lattice, similar to graphene but with a doubled lattice constant. As a consequence, the carbon atoms are separated into isolated six-membered rings, as shown in the righthand image of Fig. 2(a). Corresponding to the 0A-7B N-N pair, the ordered nitrogen-doped graphene structure is a $\sqrt{13} \times \sqrt{13}-\mathrm{R} 46.1^{\circ}$ superstructure $\left(\mathrm{C}_{12} \mathrm{~N}\right)$, as shown in Fig. 2(b). In this superstructure, the carbon atoms are connected by carbon-carbon bonds to form a single carbon domain, in contrast to the $\mathrm{C}_{3} \mathrm{~N}$ case in Fig. 2(a).

To examine the stability of the $\mathrm{C}_{3} \mathrm{~N}$ and $\mathrm{C}_{12} \mathrm{~N}$ superstructures, we use the cluster-expansion method [31] and make a direct comparison of total energies between different superstructures. In the cluster-expansion method, the alloy Hamiltonian is mapped onto a generalized Ising Hamiltonian. In brief, for the nitrogen-doped graphene configurations generated by ATAT [32], we perform spinpolarized DFT calculations to relax the cell and internal atomic coordinates. The energies of the relaxed structures are then used to extract the interaction parameters of the alloy Hamiltonian. After obtaining the interaction parameters of the alloy Hamiltonian (see the Supplemental Materials at the link in [35]), we find that the $\mathrm{C}_{3} \mathrm{~N}$ superstructure shown in Fig. 2(a) is the most stable structure among the $\mathrm{C}_{x} \mathrm{~N}$ alloys with the $25 \%$ nitrogen concentration (at least for cells with no more than 32 atoms). For the $\mathrm{C}_{12} \mathrm{~N}$ superstructure with the $0 \mathrm{~A}-7 \mathrm{~B} \mathrm{~N}-\mathrm{N}$ pairs, the relatively long-range $(5.11 \AA$ ) pair interaction is not included in the alloy Hamiltonian. Therefore, we generate all superstructures with no more than 26 atoms and the same nitrogen concentration $(7.7 \%)$ as $\mathrm{C}_{12} \mathrm{~N}$ by using the linearscaling algorithm recently proposed by Hart and Forcade [36]. Our calculations show that the $\mathrm{C}_{12} \mathrm{~N}$ superstructure shown in Fig. 2(b) has the lowest energy among all the considered structures. The lattice-dynamics calculation shows that both $\mathrm{C}_{3} \mathrm{~N}$ and $\mathrm{C}_{12} \mathrm{~N}$ have no phonons of imaginary frequencies and thus are stable [35]. Finitetemperature first-principles molecular-dynamic simulations of up to $500 \mathrm{~K}$ are performed to check the stability

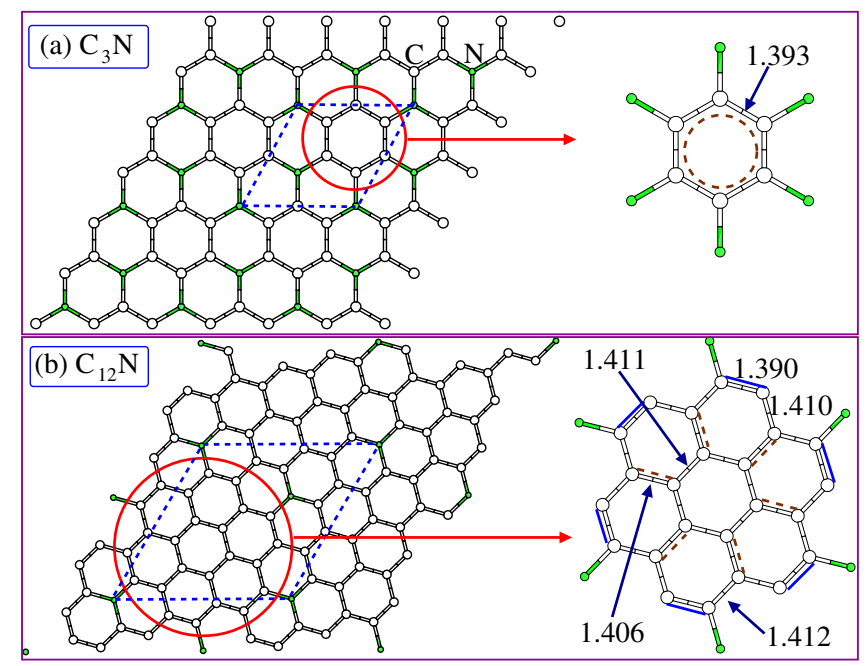

FIG. 2. (a) The ordered N-doped graphene superstructure for $\mathrm{C}_{3} \mathrm{~N}$. (b) The ordered $\mathrm{N}$-doped graphene superstructure for $\mathrm{C}_{12} \mathrm{~N}$. A unit cell of the each superstructure is enclosed by dashed lines. Each right panel displays the enlarged view of the structure that is enclosed by a red circle in the left panels. The numbers denote the bond lengths (in $\AA$ ). The Clar formulas are specified by the circle, solid, and dashed lines. 
of $\mathrm{C}_{3} \mathrm{~N}$ and $\mathrm{C}_{12} \mathrm{~N}$. We find that the $\mathrm{N}$ atoms only vibrate around the equilibrium position, which confirms the thermal stability of $\mathrm{C}_{3} \mathrm{~N}$ and $\mathrm{C}_{12} \mathrm{~N}$.

So far, we have proposed two possible stable, ordered nitrogen-carbon alloys $\left(\mathrm{C}_{3} \mathrm{~N}\right.$ and $\mathrm{C}_{12} \mathrm{~N}$, shown in Fig. 2) by considering only the graphene-based structures. In this part, we perform PSO global-minimum optimization to confirm the proposed structures. In the 2D PSO simulations [33], the graphene lattice structure is not assumed. Instead, we generate random structures (both atomic positions and cell parameters) to initialize the simulations. The initial structures are subsequently relaxed before we perform the PSO operations. The population size is set to 30 . We consider all possible cell sizes with a total number of atoms of no more than 26. The number of generations (iterations) is fixed at 30 . Our simulations show that the most stable 2D nitrogen-carbon alloy structure with $25 \% \mathrm{~N}$ concentration is indeed the structure (a p6mm plane group of 2D systems) shown in Fig. 2(a) [see also Fig. 3(a)]. The second-lowestenergy 2D structure, with the plane group p2mg [Fig. 3(b)], has a higher energy by $0.07 \mathrm{eV} / \mathrm{N}$. It should be noted that both the p2mg and p6mm structures can be viewed as nitrogen-doped graphene structures although the graphene honeycomb lattice is not assumed in the PSO simulations. The PSO simulations for the 2D nitrogen-carbon alloy structure with $7.7 \%$ nitrogen concentration do not give structures more stable than the $\mathrm{C}_{12} \mathrm{~N}$ shown in Fig. 2(b). This result supports the stability of the proposed $\mathrm{C}_{12} \mathrm{~N}$ structure. We note that the formation energies of $\mathrm{C}_{3} \mathrm{~N}$ and $\mathrm{C}_{12} \mathrm{~N}$ are positive if graphene and the $\mathrm{N}_{2}$ molecule are taken as the references. However, many experiments $[20,28]$ have shown that $\mathrm{N}$ atoms can be incorporated into graphene. What we have demonstrated is that $\mathrm{C}_{3} \mathrm{~N}$ and $\mathrm{C}_{12} \mathrm{~N}$ are the most stable structures once $\mathrm{N}$ atoms are incorporated into graphene, as can be seen from the convex hull plot in the Supplemental Materials at the link in [35].

The band structures for the $\mathrm{C}_{3} \mathrm{~N}$ and $\mathrm{C}_{12} \mathrm{~N}$ superstructures from the LDA calculations are shown in Figs. 4(a)

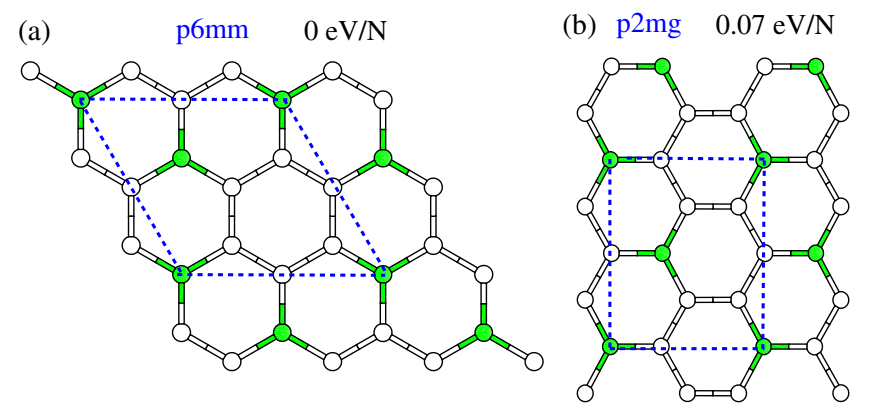

FIG. 3. (a) The most stable (lowest-energy) 2D structure (plane group: p6mm) of $\mathrm{C}_{3} \mathrm{~N}$ from the 2D PSO simulations. (b) The second-lowest-energy 2D structure (plane group: $\mathrm{p} 2 \mathrm{mg}$ ) of $\mathrm{C}_{3} \mathrm{~N}$ from the 2D PSO simulations. Dashed lines enclose the unit cells of the superstructures. The numbers denote the relative energies.
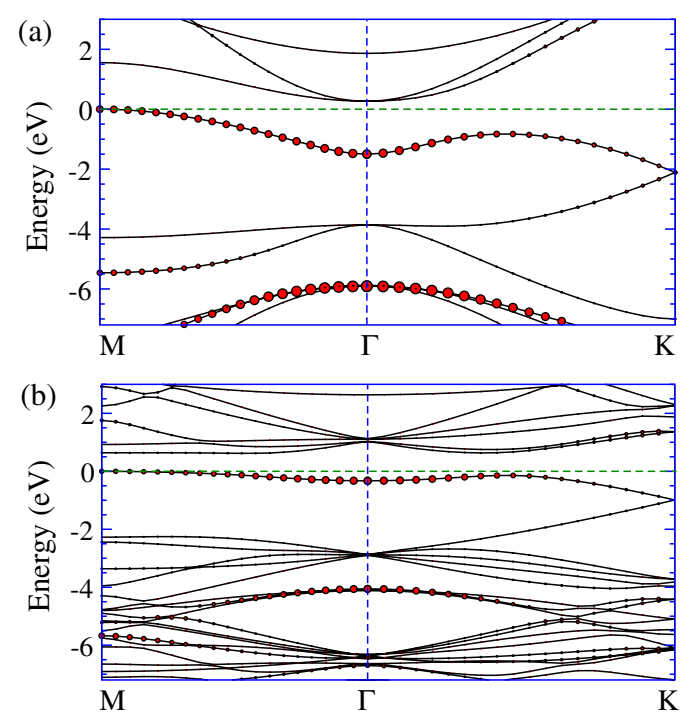

FIG. 4. The LDA band structures for (a) $\mathrm{C}_{3} \mathrm{~N}$ and (b) $\mathrm{C}_{12} \mathrm{~N}$. The horizontal green dashed lines denote the VBM levels. The contributions from $\mathrm{N} p_{z}$ orbitals to the wave functions are indicated by the radii of the solid spheres.

and 4(b), respectively. Interestingly, both superstructures are found to be semiconductors: $\mathrm{C}_{3} \mathrm{~N}$ has an indirect band gap (about $0.26 \mathrm{eV}$ from the LDA calculation) with the $\mathrm{VBM}$ at $\mathrm{M}$ and $\mathrm{CBM}$ at $\Gamma$, respectively. $\mathrm{C}_{12} \mathrm{~N}$ has instead a direct band gap (about $0.62 \mathrm{eV}$ from the LDA calculation) at the $\mathrm{M}$ point. Because the $p_{z}$ orbital of a nitrogen atom will be almost fully occupied by the two $\pi$ electrons, the mechanism for the band gap opening in graphene induced by nitrogen doping can be understood by examining only the bonding situation of carbon $\pi$ electrons. Recently, Clar's theory of the aromatic sextet [37,38] has been shown to be a simple and powerful tool to predict the stability and the electronic/magnetic structure of graphene-related systems. According to Clar's rule, for a given system, the representation with a maximum number of Clar sextets, called the Clar formula, is the most representative one. Here, we show the Clar formulas for $\mathrm{C}_{3} \mathrm{~N}$ and $\mathrm{C}_{12} \mathrm{~N}$ in the right panels of Figs. 2(a) and 2(b), respectively. In $\mathrm{C}_{3} \mathrm{~N}$, each six-membered ring forms a Clar sextet. $\mathrm{C}_{3} \mathrm{~N}$ has one unique Clar formula, and thus belongs to the class $1 \mathrm{CF}$ of the (pseudo)-all-benzenoid structure. In contrast, $\mathrm{C}_{12} \mathrm{~N}$ has no Clar sextets, and thus is nonbenzenoid. Because each nitrogen atom forms three single bonds with the neighboring carbon atoms, the nitrogen atoms are excluded when we construct the Clar formula. We note that, in the Clar formula for the -NH-terminated graphene nanoribbon, the two N-C bonds were also treated as single bonds [39]. The Clar formulas shown in Fig. 2 are in good agreement with the $\mathrm{C}-\mathrm{C}$ bond lengths of relaxed structures from the DFT calculation. All the $\mathrm{C}-\mathrm{C}$ bond lengths in $\mathrm{C}_{3} \mathrm{~N}$ are the same: $1.393 \AA$. For $\mathrm{C}_{12} \mathrm{~N}$, some C-C bonds (marked by an additional solid line) have shorter bond lengths, $1.388 \AA$, which can be considered a normal C-C double bond; and there are 
weak C-C double bonds (marked by an additional dashed line) with bond lengths of $1.406 \AA$; all the other $\mathrm{C}$ - $\mathrm{C}$ bonds are single bonds. Wassmann et al. [38] showed that all graphene-related structures have a relatively large band gap except for the (pseudo)-all-benzenoid structure with class $\mathrm{nCF}(n>=3)$, such as graphene, which has a small or zero band gap. Therefore, we expect that there is a large band gap between the occupied carbon $\pi$ states and the unoccupied carbon $\pi$ states in both $\mathrm{C}_{3} \mathrm{~N}$ and $\mathrm{C}_{12} \mathrm{~N}$. Our analysis for the wave-function characters indicates that the highest occupied band near $\Gamma$ is mainly contributed by nitrogen $p_{z}$ orbitals, and there are some contributions from carbon $p_{z}$ orbitals when the Brillouin-zone boundary is approached. The lowest unoccupied band is mostly contributed by the carbon $p_{z}$ orbitals. The gap between the highest occupied carbon $\pi$ state and the CBM is larger than $1.5 \mathrm{eV}$ (in LDA) in both $\mathrm{C}_{3} \mathrm{~N}$ and $\mathrm{C}_{12} \mathrm{~N}$, which is in accord with Clar's theory. The small band gaps for $\mathrm{C}_{3} \mathrm{~N}$ and $\mathrm{C}_{12} \mathrm{~N}$ are due to the presence of the nitrogen $p_{z}$ state (VBM state) above the highest occupied carbon $\pi$ state. $\mathrm{C}_{3} \mathrm{~N}$ has a smaller band gap because the direct hopping between $\mathrm{N} p_{z}$ orbitals in $\mathrm{C}_{3} \mathrm{~N}$ leads to the larger dispersion of the highest occupied band. We note that the dilute limit of nitrogen substitutional doping in graphene has been experimentally studied by Zhao et al. [28], where the extra electron of the nitrogen dopant was found to be delocalized into the neighboring graphene lattice, resulting in the n-type behavior. In this work, we predict that collective nitrogen doping can induce exotic semiconducting behavior in graphene. Experimentally, some disorder in the nitrogen distribution might occur due to thermal fluctuations. To see the effect of the disorder on the band gap, we have simulated a disordered configuration with $12.5 \% \mathrm{~N}$ concentration by using a 128 -atom special quasirandom structure [40]. The LDA band gap is found to be $0.08 \mathrm{eV}$. The real band gap should be larger because the LDA functional underestimates the band gap. This suggests that the disorder might change the magnitude of the band gap but should not close it. Moreover, because the ordered $\mathrm{C}_{3} \mathrm{~N}$ and $\mathrm{C}_{12} \mathrm{~N}$ are more stable at low temperatures than the disordered configuration, we believe that a reasonably large band gap should exist in nitrogen-doped graphene synthesized at low temperatures.

It is well known that LDA seriously underestimates the band gap of semiconductors; thus, we calculate the electronic structures of $\mathrm{C}_{3} \mathrm{~N}$ and $\mathrm{C}_{12} \mathrm{~N}$ by employing the screened Heyd-Scuseria-Ernzerhof 06 (HSE06) hybrid functional technique [41-43], which has been shown to give a good band gap for many semiconductors including graphene-related $\pi$ systems [44]. Our HSE06 calculations show that $\mathrm{C}_{3} \mathrm{~N}$ and $\mathrm{C}_{12} \mathrm{~N}$ have the band gaps of $0.96 \mathrm{eV}$ and $0.98 \mathrm{eV}$, respectively. We note that the band gaps of the nitrogen-doped graphene are close to the band gap (about $1.12 \mathrm{eV}$ ) in silicon. This finding suggests that $\mathrm{C}_{3} \mathrm{~N}$ and $\mathrm{C}_{12} \mathrm{~N}$ with similar electronic properties as silicon might be promising electronic materials for the next-generation complementary metal-oxide-semiconductor-device (CMOS) technology.

\section{Potential organic solar cell}

The fact that $\mathrm{C}_{12} \mathrm{~N}$ has a direct band gap of $0.98 \mathrm{eV}$ suggests that $\mathrm{C}_{12} \mathrm{~N}$ might be a promising solar-cell absorption material. To investigate this possibility, we calculate the imaginary part of the frequency-dependent dielectric function via summation over pairs of occupied and empty states without considering the local field effects, by using the HSE06 screened hybrid function. Figure 5(a) shows the calculated result $\left(\epsilon_{x x}^{2}\right)$ for one of the diagonal parts of the in-plane components. We can see a very strong peak at around $1.0 \mathrm{eV}$ arising from the interband VBM-CBM transition. Such a strong adsorption peak arises from the fact that both the VBM band and the CBM band around $\mathrm{M}$ are rather flat. Because of the dispersion of the VBM band and CBM band at other $\mathrm{K}$-points, there is also substantial optical absorption up to $1.6 \mathrm{eV}$. The absorption between $1.9 \mathrm{eV}$ and $4.0 \mathrm{eV}$ is rather weak, indicating that a solar cell with only $\mathrm{C}_{12} \mathrm{~N}$ might not be efficient because the solar energy between $1.9 \mathrm{eV}$ and $4.0 \mathrm{eV}$ may not be absorbed completely. Our HSE06 calculation shows that $\mathrm{C}_{12} \mathrm{~N}$ has a relatively high VBM level ( $-3.70 \mathrm{eV}$ with respect to the vacuum level). This suggests that $\mathrm{C}_{12} \mathrm{~N}$ can act as a donor. If an appropriate acceptor material can be found, we can construct a photovoltaic system with an architecture similar to that of the P3HT $/ \mathrm{C}_{60}-\mathrm{PCBM}$ bulk-heterojunction solar cell. We find from HSE06 calculations that the VBM and CBM levels of 6-AGNR are $-4.67 \mathrm{eV}$ and $-3.15 \mathrm{eV}$, respectively. Therefore, the band alignment between $\mathrm{C}_{12} \mathrm{~N}$ and 6-AGNR is of type-II, as shown in Fig. 5(b). In this proposed $\mathrm{C}_{12} \mathrm{~N} / 6$-AGNR solar cell, $\mathrm{C}_{12} \mathrm{~N}$ can absorb the solar energy between $0.98 \mathrm{eV}$ and approximately $1.50 \mathrm{eV}$. Also, 6-AGNR can absorb the solar energy above the band gap of $1.52 \mathrm{eV}$. The electron-hole pair excited by photons can be separated by the field due to the type-II band alignment.
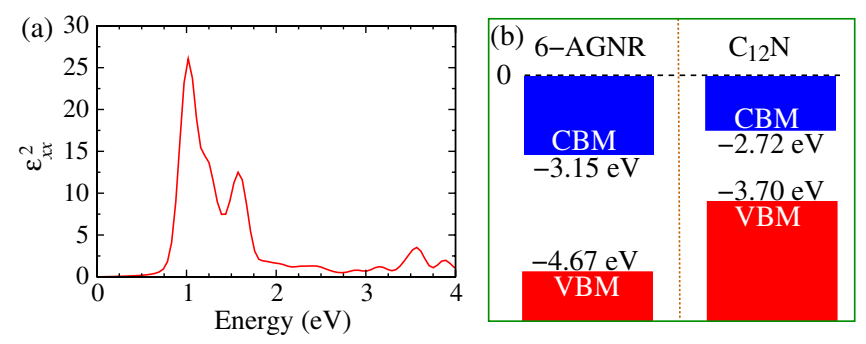

FIG. 5. (a) The computed imaginary part of the frequencydependent dielectric function from the screened exchange HSE06 calculation. (b) The band offset between $\mathrm{C}_{12} \mathrm{~N}$ and 6-AGNR from the HSE06 calculations. The numbers are the VBM and CBM levels with respect to the vacuum level. 


\section{CONCLUSION}

In summary, we have studied the interaction between substitutional nitrogen atoms in graphene and found that the nearest-neighbor interaction between nitrogen dopants is highly repulsive because of the strong electrostatic repulsion between nitrogen atoms. Our calculations reveal two stable, ordered structures of $\mathrm{N}$-doped graphene, $\mathrm{C}_{3} \mathrm{~N}$ and $\mathrm{C}_{12} \mathrm{~N}$. Both structures are semiconducting, in contrast to the common belief that nitrogen atoms simply donate electrons to the graphene Dirac cone. In particular, $\mathrm{C}_{12} \mathrm{~N}$ has a direct band gap of $0.98 \mathrm{eV}$. The substitutional nitrogen raises the VBM and CBM levels in N-doped graphene, leading to a type-II band alignment between $\mathrm{C}_{12} \mathrm{~N}$ and 6-AGNR. We propose that $\mathrm{C}_{12} \mathrm{~N} / 6$-AGNR is a promising candidate for a new type of organic solar cell with $\mathrm{C}_{12} \mathrm{~N}$ as a donor and 6-AGNR as an acceptor. Our study suggests that nitrogen doping might be a promising way to open a band gap in graphene for useful applications in electronics and photovoltaics.

\section{ACKNOWLEDGMENTS}

Work at Fudan was partially supported by the National Science Foundation of China, Pujiang plan, and The Program for Professor of Special Appointment at Shanghai Institutions of Higher Learning. Work at NREL was supported by the LDRD program funded by the U.S. Department of Energy, under Contract No. DE-AC3608GO28308.

[1] K. S. Novoselov, A. K. Geim, S. V. Morozov, D. Jiang, M. I. Katsnelson, I. V. Grigorieva, S. V. Dubonos, and A. A. Firsov, Two-Dimensional Gas of Massless Dirac Fermions in Graphene, Nature (London) 438, 197 (2005).

[2] K. S. Novoselov, E. McCann, S. V. Morozov, V. I. Falko, M. I. Katsnelson, U. Zeitler, D. Jiang, F. Schedin, and A. K. Geim, Unconventional Quantum Hall Effect and Berry's Phase of $2 \pi$ in Bilayer Graphene, Nature Phys. 2, 177 (2006).

[3] Y. Zhang, Y.-W. Tan, H.L. Stormer, and P. Kim, Experimental Observation of the Quantum Hall Effect and Berry's Phase in Graphene, Nature (London) 438, 201 (2005).

[4] S. Y. Zhou, G.-H. Gweon, A. V. Fedorov, P. N. First, W. A. De Heer, D.-H. Lee, F. Guinea, A. H. Castro Neto, and A. Lanzara, Substrate-Induced Bandgap Opening in Epitaxial Graphene, Nature Mater. 6, 770 (2007); F. Varchon, R. Feng, J. Hass, X. Li, B. Ngoc Nguyen, C. Naud, P. Mallet, J. Y. Veuillen, C. Berger, E. H. Conrad, and L. Magaud, Electronic Structure of Epitaxial Graphene Layers on SiC: Effect of the Substrate, Phys. Rev. Lett. 99, 126805 (2007).

[5] E. Rotenberg, A. Bostwick, T. Ohta, J. L. McChesney, T. Seyller, and K. Horn, Origin of the Energy Bandgap in Epitaxial Graphene, Nature Mater. 7, 258 (2008); S. Y. Zhou, D. A. Siegel, A. V. Fedorov, F.E. Gabaly, A. K.
Schmid, A.H.C. Neto, D.H. Lee, and A. Lanzara, Origin of the Energy Bandgap in Epitaxial GrapheneReply, Nature Mater. 7, 259 (2008).

[6] L. Yang, C. H. Park, Y.W. Son, M. L. Cohen, and S. G. Louie, Quasiparticle Energies and Band Gaps in Graphene Nanoribbons, Phys. Rev. Lett. 99, 186801 (2007).

[7] Y. Zhang, T. T. Tang, C. Girit, Z. Hao, M. A. Martin, A. Zettl, M. F. Crommie, Y.R. Shen, and F. Wang, Direct Observation of a Widely Tunable Bandgap in Bilayer Graphene, Nature (London) 459, 820 (2009).

[8] E. V. Castro, K. S. Novoselov, S. V. Morozov, N. M. R. Peres, J. M. B. Lopes dos Santos, Johan Nilsson, F. Guinea, A. K. Geim, and A.H. Castro Neto, Biased Bilayer Graphene: Semiconductor with a Gap Tunable by the Electric Field Effect, Phys. Rev. Lett. 99, 216802 (2007).

[9] E. Bekyarova, M.E. Itkis, P. Ramesh, C. Berger, M. Sprinkle, W. A. de Heer, and R.C. Haddon, Chemical Modification of Epitaxial Graphene: Spontaneous Grafting of Aryl Groups, J. Am. Chem. Soc. 131, 1336 (2009).

[10] S. Sarkar, E. Bekyarova, S. Niyogi, and R. C. Haddon, Diels-Alder Chemistry of Graphite and Graphene: Graphene as Diene and Dienophile, J. Am. Chem. Soc. 133, 3324 (2011).

[11] S. Ryu, M. Y. Han, J. Maultzsch, T. F. Heiz, P. Kim, M. L. Steigerwald, and L.E. Brus, Reversible Basal Plane Hydrogenation of Graphene, Nano Lett. 8, 4597 (2008).

[12] H. J. Xiang, E. J. Kan, S.-H. Wei, M.-H. Whangbo, and J. L. Yang, "Narrow" Graphene Nanoribbons Made Easier by Partial Hydrogenation, Nano Lett. 9, 4025 (2009).

[13] H. J. Xiang, E. J. Kan, S.-H. Wei, X. G. Gong, and M.-H. Whangbo, Thermodynamically Stable Single-Side Hydrogenated Graphene, Phys. Rev. B 82, 165425 (2010).

[14] D.W. Boukhvalov and M.I. Katsnelson, Chemical Functionalization of Graphene, J. Phys. Condens. Matter 21, 344205 (2009).

[15] J. O. Sofo, A. S. Chaudhari, and G. D. Barber, Graphane: A Two-Dimensional Hydrocarbon, Phys. Rev. B 75, 153401 (2007).

[16] J. Zhou, Q. Wang, Q. Sun, X. S. Chen, Y. Kawazoe, and P. Jena, Ferromagnetism in Semihydrogenated Graphene Sheet, Nano Lett. 9, 3867 (2009).

[17] N. Lu, Z. Li, and J. L. Yang, Electronic Structure Engineering via On-Plane Chemical Functionalization: A Comparison Study on Two-Dimensional Polysilane and Graphane, J. Phys. Chem. C 113, 16741 (2009).

[18] S. Lebégue, M. Klintenberg, O. Eriksson, and M. I. Katsnelson, Accurate Electronic Band Gap of Pure and Functionalized Graphane from GW Calculations, Phys. Rev. B 79, 245117 (2009).

[19] H. J. Xiang, S.-H. Wei, and X. G. Gong, Structural Motifs in Oxidized Graphene: A Genetic Algorithm Study Based on Density Functional Theory, Phys. Rev. B 82, 035416 (2010).

[20] X. Wang, X. Li, L. Zhang, Y. Yoon, P. K. Weber, H. Wang, J. Guo, and H. Dai, N-Doping of Graphene through Electrothermal Reactions with Ammonia, Science 324, 768 (2009). 
[21] S. Yu, W. Zheng, C. Wang, and Q. Jiang, Nitrogen/Boron Doping Position Dependence of the Electronic Properties of a Triangular Graphene, ACS Nano 4, 7619 (2010).

[22] Y. Li, Z. Zhou, P. Shen, and Z. Chen, Spin Gapless Semiconductor-Metal-Half-Metal Properties in NitrogenDoped Zigzag Graphene Nanoribbons, ACS Nano 3, 1952 (2009).

[23] B. Biel, X. Blase, F. Triozon, and S. Roche, Anomalous Doping Effects on Charge Transport in Graphene Nanoribbons, Phys. Rev. Lett. 102, 096803 (2009).

[24] A. Lherbier, X. Blase, Y.-M. Niquet, F. Triozon, and S. Roche, Charge Transport in Chemically Doped 2D Graphene, Phys. Rev. Lett. 101, 036808 (2008).

[25] B. Huang, Electronic Properties of Boron and Nitrogen Doped Graphene Nanoribbons and Its Application for Graphene Electronics, Phys. Lett. A 375, 845 (2011).

[26] Dehui Deng, Xiulian Pan, Liang Yu, Yi Cui, Yeping Jiang, Jing Qi, Wei-Xue Li, Qiang Fu, Xucun Ma, Qikun Xue, Gongquan Sun, and Xinhe Bao, Toward N-Doped Graphene via Solvothermal Synthesis, Chem. Mater. 23, 1188 (2011).

[27] H. Liu, Y. Liu, and D. Zhu, Chemical Doping of Graphene, J. Mater. Chem. 21, 3335 (2011).

[28] Liuyan Zhao, Rui He, Kwang Taeg Rim, Theanne Schiros, Keun Soo Kim, Hui Zhou, Christopher Gutiérrez, S. P. Chockalingam, Carlos J. Arguello, Lucia Pálová et al., Visualizing Individual Nitrogen Dopants in Monolayer Graphene, Science 333, 999 (2011).

[29] P.E. Blöchl, Projector Augmented-Wave Method, Phys. Rev. B 50, 17953 (1994); G. Kresse and D. Joubert, From Ultrasoft Pseudopotentials to the Projector AugmentedWave Method, ibid. 59, 1758 (1999).

[30] G. Kresse and J. Furthmüller, Efficiency of Ab-Initio Total Energy Calculations for Metals and Semiconductors Using a Plane-Wave Basis Set, Comput. Mater. Sci. 6, 15 (1996); Efficient Iterative Schemes for Ab Initio TotalEnergy Calculations Using a Plane-Wave Basis Set, Phys. Rev. B 54, 11169 (1996).

[31] L. G. Ferreira, S.-H. Wei, and A. Zunger, First-Principles Calculation of Alloy Phase Diagrams: The RenormalizedInteraction Approach, Phys. Rev. B 40, 3197 (1989).

[32] A. van de Walle, M. Asta, and G. Ceder, The Alloy Theoretic Automated Toolkit: A User Guide, CALPHAD: Comput. Coupling Phase Diagrams Thermochem. 26, 539 (2002) [http://www.its.caltech.edu/ avdw/atat/].
[33] Xinyu Luo, Jihui Yang, Hanyu Liu, Xiaojun Wu, Yanchao Wang, Yanming Ma, Su-Huai Wei, Xingao Gong, and H. J. Xiang, Predicting Two-Dimensional Boron-Carbon Compounds by the Global Optimization Method, J. Am. Chem. Soc. 133, 16285 (2011).

[34] Y. Wang, J. Lv, L. Zhu, and Y. Ma, Crystal Structure Prediction via Particle-Swarm Optimization, Phys. Rev. B 82, 094116 (2010) [http://nlshm-lab.jlu.edu.cn/ calypso .html].

[35] See the Supplemental Materials at http://link.aps.org/ supplemental/10.1103/PhysRevX.2.011003 for the clusterexpansion parameters, the convex hull of the nitrogencarbon compounds, and phonon dispersions of $\mathrm{C}_{3} \mathrm{~N}$ and $\mathrm{C}_{12} \mathrm{~N}$.

[36] G. L. W. Hart and R.W. Forcade, Generating Derivative Structures from Multilattices: Algorithm and Application to Hcp Alloys, Phys. Rev. B 80, 014120 (2009).

[37] E. Clar, The Aromatic Sextet (Wiley, London, 1972).

[38] T. Wassmann, A. P. Seitsonen, A. M. Saitta, M. Lazzeri, and F. Mauri, Clar's Theory, $\pi$-Electron Distribution, and Geometry of Graphene Nanoribbons, J. Am. Chem. Soc. 132, 3440 (2010).

[39] A. P. Seitsonen, A. M. Saitta, T. Wassmann, M. Lazzeri, and F. Mauri, Structure and Stability of Graphene Nanoribbons in Oxygen, Carbon Dioxide, Water, and Ammonia, Phys. Rev. B 82, 115425 (2010).

[40] A. Zunger, S.-H. Wei, L. G. Ferreira, and J. E. Bernard, Special Quasirandom Structures, Phys. Rev. Lett. 65, 353 (1990); S.-H. Wei, L. G. Ferreira, J. E. Bernard, and A. Zunger, Electronic Properties of Random Alloys: Special Quasirandom Structures, Phys. Rev. B 42, 9622 (1990).

[41] J. Heyd, G.E. Scuseria, and M. Ernzerhof, Hybrid Functionals Based on a Screened Coulomb Potential, J. Chem. Phys. 118, 8207 (2003).

[42] A. V. Krukau, O. A. Vydrov, A. F. Izmaylov, and G. E. Scuseria, Influence of the Exchange Screening Parameter on the Performance of Screened Hybrid Functionals, J. Chem. Phys. 125, 224106 (2006).

[43] J. Paier, M. Marsman, K. Hummer, G. Kresse, I. C. Gerber, and J. G. Ángyán, Screened Hybrid Density Functionals Applied to Solids, J. Chem. Phys. 124, 154709 (2006).

[44] O. Hod, V. Barone, and G. E. Scuseria, Half-Metallic Graphene Nanodots: A Comprehensive First-Principles Theoretical Study, Phys. Rev. B 77, 035411 (2008). 